\title{
Nonsynonymous T280M gene variant of CX3CR1 in South Indian population is associated with reduced risk for vascular disease in patients with diabetes mellitus
}

\author{
S Sumi $\mathrm{PhD}^{1 *}$, Surya Ramachandran $\mathrm{PhD}^{1 *}$, V Raman Kutty MD MPhil MPH${ }^{2}$, Maulin M Patel MSc ${ }^{1}$, \\ TN Anand $\mathrm{MPH}^{2}$, Ajit Mullassari MD DM³ ${ }^{3}$ CC Kartha MD FRCP ${ }^{1}$
}

\begin{abstract}
S Sumi, S Ramachandran, VR Kutty, et al. Nonsynonymous T280M gene variant of CX3CR1 in South Indian population is associated with reduced risk for vascular disease in patients with diabetes mellitus. Curr Res Cardiol 2015;2(4):188-192.
\end{abstract}

BACKGROUND: Vascular inflammation leading to coronary artery disease (CAD) is a major complication of type 2 diabetes mellitus (T2DM). The chemokine receptor CX3CR1 is a key regulator in vascular injuryrelated inflammation.

OBJECTIVE: The authors examined the T280M and V249I gene variants of the CX3CR1 gene in patients with T2DM, CAD and CAD associated with T2DM, to understand the effect of these polymorphisms on the disease phenotype.

METHODS: Whole blood samples were collected from 913 South Indian subjects comprising 160 patients with T2DM, 284 with T2DM and CAD, 198 with CAD and 271 healthy subjects. T280M and V249I variants of the CX3CR1 gene were genotyped in these study subjects using polymerase chain reaction (PCR)-restriction fragment length polymorphism analysis and PCR-DNA sequencing.

$\mathrm{D}$ iabetes mellitus and vascular diseases represent two faces of the same coin because they share several underlying genetic and environmental risk factors (1). Coronary artery disease (CAD) is a serious cardiac complication and primary cause of death in patients with type 2 diabetes mellitus (T2DM) (2). Despite evidence that susceptibility to CAD has an extensive genetic basis, mapping out the genetic landscape has been unsuccessful to date due to the multifactorial nature of the disease $(3,4)$. The CAD-T2DM phenotype is further complex. It is possible that there exists a shared genetic background underlying but specific to diabetic vascular disease. Identifying gene variants in this milieu from the existing key players would help us to better understand the initiation and progression of vascular complications in diabetes.

Fractalkine (FKN) ligand-CX3CR1 receptor signalling modulates monocyte adhesion to endothelial cells and adipocytes. Enhanced FKN-CX3CR1 binding is associated with obesity, insulin resistance and pathogenesis of CAD (5). Two polymorphisms, T280M and V249I, present in the open reading frame of the CX3CR1 gene, result in decreased FKN/CX3CR1 signalling and reduced susceptibility to CAD. These polymorphisms are considered to be 'atheroprotective' in various cohorts $(6,7)$.

On the other hand, FKN signalling is also a key regulator of pancreatic islet beta cell survival and insulin secretion (8). A reduced FKN/CX3CR1 interaction is a significant event underlying beta cell dysfunction and impaired insulin secretion. Because V249I and T280M nonsynonymous polymorphisms of the CX3CR1 gene lead to reduced binding and signalling, it is possible that individuals who

\begin{abstract}
RESULTS: Multivariate logistic regression analysis revealed that the frequencies of 280M and 249I alleles were higher in controls than in cases in both the CAD and T2DM groups. Adjusted odds of T280M according to logistic regression were significant in all disease groups compared withhealthy subjects. Both MM and II genotypes of T280M and V249I polymorphisms were found to have an atheroprotective role in CAD and T2DM. The association of these polymorphisms were then examined in patients with $\mathrm{CAD}$ who were also diagnosed with diabetes. The $\mathrm{M}$ allele of T280M polymorphism was associated with attenuated risk for CAD and T2DM.

CONCLUSIONS: The present study demonstrated a protective role of the T280M variant of CX3CR1 gene for vascular complication in patients with T2DM. This polymorphism may reduce the binding strength of the chemokine receptor, thus attenuating inflammation both in vascular wall and adipose tissue.
\end{abstract}

Key Words: Chemokine; Coronary artery disease; Polymorphism; Type 2 diabetes mellitus

harbour these polymorphisms may be genetically susceptible to diabetes. CX3CR1 has also been implicated as an adipose tissue chemokine contributing to adipose inflammation leading to the T2DM phenotype (5). We analyzed the prevalence of these two variants in patients with T2DM with and without CAD to understand the association of these polymorphisms with risk for vascular complications in patients with diabetes mellitus.

The investigation was a hospital-based, case-control study consisting of 913 subjects. The subjects were of Dravidian origin belonging to South India, among whom a high prevalence of diabetes mellitus has been reported $(9,10)$. The prevalence of T280M and V249I variants of CX3CR1 gene is unknown in this population.

Study subjects and groups

\section{METHODS}

Study subjects were recruited from three multispecialty centres of South India. The study was approved by institutional ethics committees of Rajiv Gandhi Centre for Biotechnology, Thiruvananthapuram and participating hospitals (Madras Medical Mission, Chennai, PRS hospital and Indian Institute of Diabetes, Thiruvananthapuram) and all study participants provided informed written consent. Subjects with hypertension, congenital heart disease, renal disease, cardiac disease other than $\mathrm{CAD}$, microvascular disease and pregnancy were excluded. Whole blood samples $(3 \mathrm{~mL})$ after an overnight fast were collected from 913 subjects (age range 24 to 93 years). The subjects were grouped into four categories: T2DM $(n=160)$; patients with T2DM and CAD $(n=284)$; patients with CAD but no T2DM

\footnotetext{
*Authors who contributed equally

${ }^{1}$ Rajiv Gandhi Centre for Biotechnology, Thiruvananthapuram; ${ }^{2}$ Achutha Menon Centre for Health Science Studies, Sree Chitra Tirunal Institute for Medical Sciences and Technology, Thiruvananthapuram, Kerala; ${ }^{3}$ Madras Medical Mission, Chennai, India

Correspondence: Dr CC Kartha, Cardiovascular Diseases and Diabetes Biology, Rajiv Gandhi Centre for Biotechnology, Thiruvananthapuram, India 695014. Telephone 91-471-2529448, fax 91-471-2529505, e-mail cckartha@rgcb.res.in
} 
TABLE 1

Baseline clinical parameters of the study population

\begin{tabular}{|c|c|c|c|c|}
\hline \multirow[b]{2}{*}{ Variable } & \multicolumn{4}{|c|}{ Group } \\
\hline & Normal & T2DM & CAD & T2DM + CAD \\
\hline \multicolumn{5}{|l|}{ Age, years } \\
\hline$>45$ & $144(53.1)$ & $136(85)$ & $172(86.9)$ & $266(93.7)$ \\
\hline$<45$ & $127(46.9)$ & $24(15)$ & $26(13.1)$ & $18(6.3)$ \\
\hline \multicolumn{5}{|l|}{ Sex } \\
\hline Male & $134(49.4)$ & $72(45)$ & $162(81.8)$ & $234(82.4)$ \\
\hline Female & $137(50.6)$ & $88(55)$ & $36(18.2)$ & $50(17.6)$ \\
\hline \multicolumn{5}{|l|}{ Smoking } \\
\hline Yes & $27(10)$ & $17(10.6)$ & $31(15.7)$ & $59(20.8)$ \\
\hline No & $244(90)$ & $143(89.4)$ & $167(84.3)$ & $225(79.2)$ \\
\hline \multicolumn{5}{|l|}{ Alcohol } \\
\hline Yes & $11(4.1)$ & $10(6.2)$ & $14(7.1)$ & $20(7)$ \\
\hline No & $260(95.9)$ & $150(93.8)$ & $184(92.9)$ & $264(93)$ \\
\hline \multicolumn{5}{|c|}{ Fasting blood glucose, $\mathrm{mmol} / \mathrm{L}$} \\
\hline$>6.94$ & $0(0)$ & $116(72.5)$ & $24(12.1)$ & $175(61.6)$ \\
\hline$\leq 6.94$ & $271(100)$ & $44(27.5)$ & 174(87.9) & $109(38.4)$ \\
\hline \multicolumn{5}{|l|}{ HbA1c, \% } \\
\hline$\geq 6.5$ & $1(0.4)$ & $147(91.9)$ & $0(0)$ & $253(89.1)$ \\
\hline$<6.5$ & $270(99.6)$ & $13(8.1)$ & $198(100)$ & $31(10.9)$ \\
\hline \multicolumn{5}{|c|}{ Cholesterol, mmol/L } \\
\hline$>5.18$ & $20(7.4)$ & $63(39.4)$ & $42(21.2)$ & $28(9.9)$ \\
\hline$\leq 5.18$ & $251(92.6)$ & $97(60.6)$ & $156(78.8)$ & $256(90.1)$ \\
\hline \multicolumn{5}{|l|}{ LDL, mmol/L } \\
\hline$>2.59$ & $5(1.8)$ & 95 (59.4) & 71 (35.9) & $52(18.3)$ \\
\hline$\leq 2.59$ & $266(98.2)$ & $65(40.6)$ & $127(64.1)$ & $232(81.7)$ \\
\hline \multicolumn{5}{|l|}{$\mathrm{HDL}, \mathrm{mmol} / \mathrm{L}$} \\
\hline$\geq 1.55$ & $2(0.7)$ & $44(27.5)$ & $96(48.5)$ & $217(76.4)$ \\
\hline $1.04-1.53$ & $183(67.5)$ & $104(65)$ & $97(49)$ & $62(21.8)$ \\
\hline$<1.04$ & $86(31.7)$ & $12(7.5)$ & $5(2.5)$ & $5(1.8)$ \\
\hline \multicolumn{5}{|c|}{ Triglycerides, $\mathrm{mmol} / \mathrm{L}$} \\
\hline$>1.7$ & $2(0.7)$ & $12(7.5)$ & $74(37.4)$ & $91(32)$ \\
\hline$\leq 1.7$ & $269(99.3)$ & $148(92.5)$ & $124(62.6)$ & $193(68)$ \\
\hline
\end{tabular}

Data presented as $n$ (\%). CAD Coronary artery disease; HbA1c Glycated hemoglobin; HDL High-density lipoprotein; T2DM Type 2 diabetes mellitus; LDL Low-density lipoprotein

$(\mathrm{n}=198)$; and healthy individuals $(\mathrm{n}=271)$. Demographic characteristics and biochemical parameters of subjects were collected and analyzed. Diabetes was assessed by measuring fasting blood glucose (FBG) and glycated hemoglobin (HbA1c) levels. CAD was detected using positive treadmill test and/or coronary angiography.

Genomic DNA extraction

Genomic DNA from peripheral blood was extracted using QIAamp DNA blood mini kit (Qiagen, USA) according to the manufacturer's instructions. Quantity and quality of DNA was measured using nanodrop-1000 spectrophotometer (Thermo Scientific, USA) at $260 \mathrm{~nm}$.

Genotyping using polymerase chain reaction-restriction fragment length polymorphism

The genotyping of CX3CR1 V249I (rs3732379) and T280M (rs3732378) polymorphisms were performed using polymerase chain reaction-restriction fragment length polymorphism (PCR-RFLP) techniques. DNA fragments containing polymorphisms were amplified using specificprimers, CX3CR1:5'-CCGAGGTCCTTCAGGAAATCT-3' and 5'-TCAGCATCAGGTTCAGGAACTC-3' (7). PCR conditions reactions were performed in a thermal cycler with an initial denaturation for $5 \mathrm{~min}$ at $94^{\circ} \mathrm{C}, 35$ cycles of denaturing at $94^{\circ} \mathrm{C}$ for $30 \mathrm{~s}$, annealing at $62^{\circ} \mathrm{C}$ for $30 \mathrm{~s}$ and extension at $72^{\circ} \mathrm{C}$ for $1 \mathrm{~min}$. A final extension at $72^{\circ} \mathrm{C}$ for $7 \mathrm{~min}$ was also performed. The PCR products were digested with BsmBI and Psp1406I enzymes (New England Biolabs, United Kingdom).
TABLE 2

Mean values in the study groups

\begin{tabular}{lcccc}
\hline Variable & $\begin{array}{c}\text { Normal } \\
(\mathbf{n}=\mathbf{2 7 1})\end{array}$ & $\begin{array}{c}\text { T2DM } \\
(\mathbf{n}=160)\end{array}$ & $\begin{array}{c}\text { CAD } \\
(\mathbf{n}=198)\end{array}$ & $\begin{array}{c}\text { T2DM + CAD } \\
(\mathbf{n}=\mathbf{2 8 4})\end{array}$ \\
\hline Age, years & $45.32 \pm 12.99$ & $55.4 \pm 10.7^{*}$ & $57.43 \pm 12.67^{*}$ & $59.29 \pm 9.66^{\star}$ \\
FBG, mmol/L & $5.28 \pm 0.55$ & $9.03 \pm 2.77^{*}$ & $5.83 \pm 0.89^{*}$ & $8.04 \pm 2.31^{*}$ \\
HbA1c, \% & $5.87 \pm 0.51$ & $8.21 \pm 1.22^{*}$ & $5.82 \pm 0.36^{\dagger}$ & $8.21 \pm 1.64^{*}$ \\
Cholesterol* $^{*}$ & $4.61 \pm 0.42$ & $5.0 \pm 0.8^{*}$ & $4.39 \pm 1.31^{\ddagger}$ & $3.91 \pm 1.05^{\star}$ \\
LDL* $^{*}$ & $2.23 \pm 0.33$ & $2.82 \pm 0.59^{*}$ & $2.66 \pm 0.89^{*}$ & $2.08 \pm 0.77^{\ddagger}$ \\
HDL* $_{\text {Triglycerides* }}$ & $1.44 \pm 0.15$ & $1.2 \pm 0.23^{*}$ & $1.08 \pm 0.28^{*}$ & $0.89 \pm 0.26^{\star}$ \\
\hline
\end{tabular}

Data presented as mean \pm SD of clinical profile of each study group. *Data presented as mmol/L. CAD Coronary artery disease; FBG Fasting blood glucose; HbA1c Glycated hemoglobin; HDL High-density lipoprotein; LDL Low-density lipoprotein; T2DM Type 2 diabetes mellitus. $P$ values calculated in comparison with control group. ${ }^{\star} P<0.001 ;{ }^{\dagger} P<0.05 ;{ }^{\ddagger} P<0.01$

TABLE 3

Frequency of genotypes in controls, and patients with coronary artery disease (CAD)

\begin{tabular}{|c|c|c|c|c|}
\hline Genotype & $\begin{array}{l}\text { Control } \\
(n=271)\end{array}$ & $\begin{array}{c}\text { CAD } \\
(n=198)\end{array}$ & OR (95\% Cl) & $\begin{array}{c}\text { Adjusted OR } \\
(95 \% \mathrm{Cl})^{*}\end{array}$ \\
\hline \multicolumn{5}{|c|}{ CX3CR1 T280M } \\
\hline $\mathrm{T} / \mathrm{T}$ & 39 (14.4) & $135(68.2)$ & 1 (reference) & 1 (reference) \\
\hline$T / M$ & $127(46.9)$ & $50(25.3)$ & $0.11(0.07-0.18)$ & $0.11(0.06-0.19)^{\dagger}$ \\
\hline $\mathrm{M} / \mathrm{M}$ & $105(38.7)$ & $13(6.6)$ & $0.04(0.02-0.07)$ & $0.02(0.01-0.06)^{\dagger}$ \\
\hline$T / M+M / M$ & $232(85.6)$ & $63(31.8)$ & $0.08(0.04-0.15)$ & $0.07(0.04-0.12)^{\dagger}$ \\
\hline \multicolumn{5}{|c|}{ CX3CR1 V249I } \\
\hline $\mathrm{V} / \mathrm{V}$ & $124(45.8)$ & 117 (59.1) & 1 (reference) & 1 (reference) \\
\hline V/I & $124(45.8)$ & 70 (35.4) & $0.59(0.40-0.89)$ & $0.60(0.38-0.94)^{\ddagger}$ \\
\hline 1/I & $23(8.5)$ & $11(5.6)$ & $0.50(0.23-1.08)$ & $0.66(0.26-1.60)$ \\
\hline$V / I+I / I$ & $147(54.2)$ & $81(40.9)$ & $0.58(0.40-0.84)$ & $0.61(0.40-0.94)^{\ddagger}$ \\
\hline
\end{tabular}

Data presented as $n$ (\%) unless otherwise indicated. *Logistic regression analysis adjusted for age, sex, cholesterol, glycated hemoglobin, smoking and alcohol. ${ }^{\dagger} P<0.01 ;{ }^{\ddagger} P<0.05$

RFLP was performed by incubating the PCR product with $1 \mu \mathrm{L}$ of restriction enzyme at $37^{\circ} \mathrm{C}$ for $2 \mathrm{~h}$, followed by inactivation of enzyme at $65^{\circ} \mathrm{C}$ for $10 \mathrm{~min}$.

PCR DNA sequencing

PCR was performed, as mentioned earlier, and products were purified using gel band elution kit (GE Healthcare, USA). DNA sequencing based on Sanger dideoxy chain termination sequencing was performed on a DNA analyzer utilizing Bigdye terminator chemistry (ABI 3100, Applied Biosystems, USA). All sequences were analyzed using Sequencing analysis version 1.0 (Applied Biosystems, USA).

Statistical analysis

Statistical tests were performed using R software on the GNU platform (R Core Team, 2013, R: A language and environment for statistical computing, R Foundation for Statistical Computing, Austria). Data regarding continuous variables were expressed as mean $\pm \mathrm{SD}$ and data of noncontinuous variables as frequency ( $\mathrm{n}[\%]$ ); $\mathrm{P}<0.05$ was considered to be statistically significant. Hardy-Weinberg equilibrium was tested for a goodness-of-fit using a $\chi^{2}$ test. The genetic variants and their risk for disease were computed according to ORs and 95\% CIs by logistic regression analysis.

\section{RESULTS}

Clinical and baseline profile of study subjects

The study population consisted of 913 subjects, which included 198 with CAD (162 men and 36 women), 160 with T2DM and no associated vascular diseases ( 72 men and 88 women) and 284 diagnosed with 

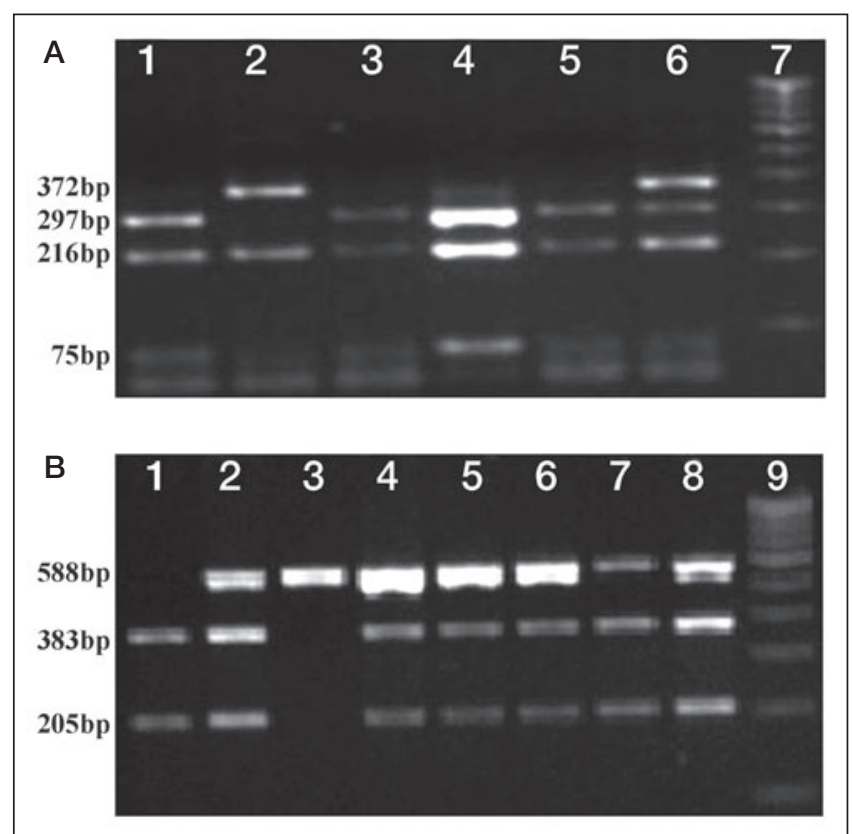

Figure 1) Gel image demonstrating polymerase chain reaction restriction fragment length polymorphism analysis of the CX3CR1 gene. A T280M polymorphism: lanes 1, 3, 4 and 5: homozygous wild (TT), lane 6: heterozygous polymorphic (TM), lane 2: homozygous polymorphic (MM), lane 7: 100 base pair (bp) DNA ladder. B V249I polymorphism: lane 1: homozygous wild (VV), lanes 2 and 4 to 8: heterozygous polymorphic (VI), lane 3: homozygous polymorphic (II), lane 9: 100 bp DNA ladder

both T2DM and CAD (234 men and 50 women); 271 normal healthy individuals (134 men and 137 women) were also included. The baseline demographic characteristics and clinical parameters of the study subjects are summarized in Table 1 .

The mean \pm SD values of each clinical parameter in the four study groups are presented in Table 2. Risk factors for vascular disease in diabetes, such as lipid profile and blood glucose levels, were markedly higher in the patient groups. In patients with T2DM with or without $\mathrm{CAD}, \mathrm{FBG}$ and HbA1c levels were elevated compared with normal individuals $(\mathrm{P}<0.05)$. Lipid profile analysis revealed significantly higher serum levels of total cholesterol, low-density lipoprotein and triglycerides, and lower high-density lipoprotein in all patient groups compared with the group of healthy volunteers $(\mathrm{P}<0.01)$. An altered lipid profile was observed in patients with T2DM who did not have $\mathrm{CAD}(\mathrm{P}<0.01)$. Lifestyle factors affecting risk for cardiovascular diseases, such as cigarette smoking and alcohol consumption, were not significantly associated with any of the patient groups.

Genotype frequencies of CX3CR1 T280M and V249I variants in disease groups

Genotype distribution of CX3CR1 V249I (rs3732379) and T280M (rs3732378) gene variants in patients with CAD and in patients with T2DM with and without CAD are shown in Tables 3 to 5. The genotype distribution of these two polymorphisms was in Hardy-Weinberg equilibrium $(\mathrm{P}>0.05)$. The results from PCR-RFLP were confirmed by PCR sequencing; the results of both genotyping techniques corroborated well. A representative gel image of these polymorphisms is presented in Figure 1. Representative electropherograms of CX3CR1 T280M and V249I polymorphisms are shown in Figure 2.

Logistic regression analysis of T280M and V249I polymorphisms revealed a reduced risk for CAD (Table 3). An OR of 0.07 (95\% CI 0.04 to 0.12 ) was observed in patients carrying TM heterozygous and MM homozygous mutant genotypes when compared with patients carrying the TT wild genotype. The genotype frequency for V249I polymorphism was also found to be significantly different between patients

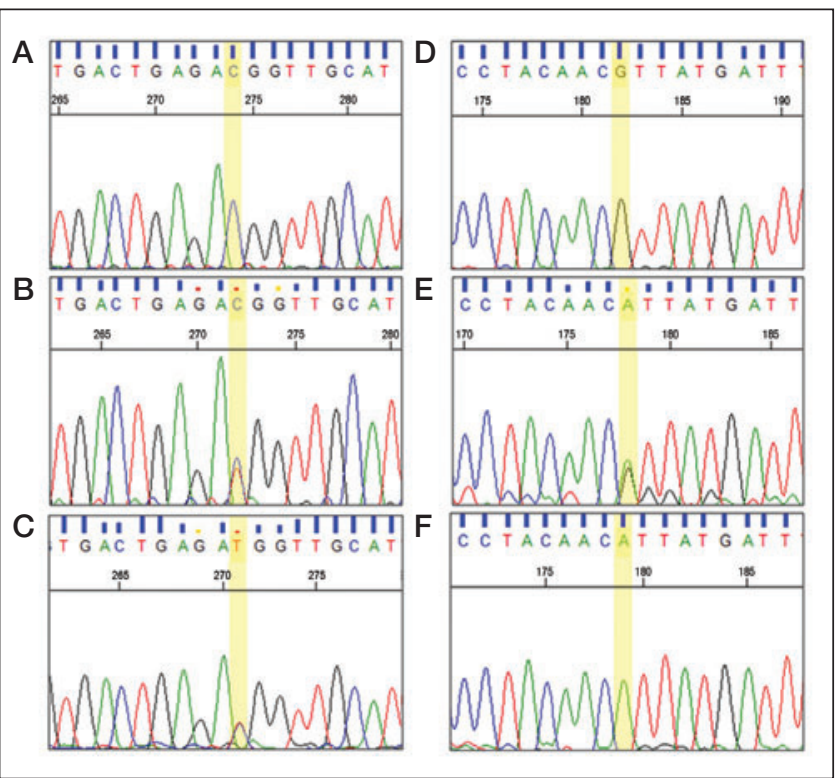

Figure 2) Sequence analysis of rs3732378 and rs37323789 in patients with type 2 diabetes mellitus and coronary artery disease. Left panel reports rs3732378: NM_001337.3:c.839C $\rightarrow$ T, the highlighted area indicates (A) C (wild type allele), (B) heterozygous C/T (C) T (polymorphic allele) of CX3CR1 gene. Right panel reports rs3732379: NM_001337.3: c.745G $\rightarrow \mathrm{A}$ (D) G (wild type allele), (E) heterozygous G/A, (F) A (polymorphic allele) of the CX3CR1 gene

\section{TABLE 4}

Frequency of genotypes in healthy controls, and patients with type 2 diabetes mellitus (T2DM)

\begin{tabular}{|c|c|c|c|c|}
\hline Genotype & $\begin{array}{l}\text { Control } \\
(n=271)\end{array}$ & $\begin{array}{c}\text { T2DM } \\
(n=160)\end{array}$ & OR $(95 \% \mathrm{Cl})$ & $\begin{array}{l}\text { Adjusted OR } \\
(95 \% \mathrm{Cl})^{*}\end{array}$ \\
\hline \multicolumn{5}{|c|}{ CX3CR1 T280M } \\
\hline$T / T$ & $39(14.4)$ & 59 (36.9) & 1.00 (reference) & 1.00 (reference) \\
\hline $\mathrm{T} / \mathrm{M}$ & $127(46.9)$ & $82(51.2)$ & $0.42(0.26-0.69)$ & $0.49(0.12-2.10)$ \\
\hline $\mathrm{M} / \mathrm{M}$ & $105(38.7)$ & 19 (11.9) & $0.11(0.06-0.22)$ & $0.10(0.01-0.60)^{\dagger}$ \\
\hline $\mathrm{T} / \mathrm{M}+\mathrm{M} / \mathrm{M}$ & 232 (85.6) & 101 (63.1) & $0.28(0.18-0.45)$ & $0.29(0.07-1.14)$ \\
\hline \multicolumn{5}{|c|}{ CX3CR1 V249I } \\
\hline $\mathrm{V} / \mathrm{V}$ & $124(45.8)$ & 70 (43.8) & 1.00 (reference) & 1.00 (reference) \\
\hline V/I & $124(45.8)$ & $76(47.5)$ & $1.08(0.72-1.63)$ & $0.58(0.15-1.93)$ \\
\hline $1 / 1$ & $23(8.5)$ & $14(8.8)$ & $1.07(0.52-2.22)$ & $0.18(0.06-1.80)$ \\
\hline$V / I+I / I$ & $147(54.2)$ & $90(56.25)$ & $1.08(0.73-1.60)$ & $0.49(0.13-1.54)$ \\
\hline
\end{tabular}

Data presented as $n$ (\%) unless otherwise indicated. ${ }^{*}$ ORs (logistic regression analysis) adjusted for age, sex, cholesterol, glycated hemoglobin, smoking and alcohol; ${ }^{\dagger} P<0.05$

and controls, with a protective OR 0.61 (95\% CI 0.40 to 0.94) for the VI heterozygous and II homozygous mutant genotypes compared with VV wild genotype. The calculated $\mathrm{OR}$ was $<1$, indicating that the II and MM genotype have a protective effect for CAD. These results were stable after adjustment for traditional risk factors (age, sex, lipid profile, HbA1c and lifestyle risk factors).

Similar results were obtained for the T2DM group. Genotype frequencies and risk estimates for T2DM are shown in Table 4. T280M polymorphism of CX3CR1 gene was associated with a reduced risk for developing diabetes in the South Indian population (OR 0.29 [95\% CI 0.07 to 1.14$]$ ). VI heterozygous and II homozygous mutant genotypes versus VV wild genotype of V249I polymorphism had an OR of 0.49 (95\% CI 0.13 to 1.54$)$.

Effect of CX3CR1 gene variants on diabetic vascular disease The CX3CR1 gene polymorphisms have been associated with a protective role for atherogenesis in patients with CAD. The analysis also 

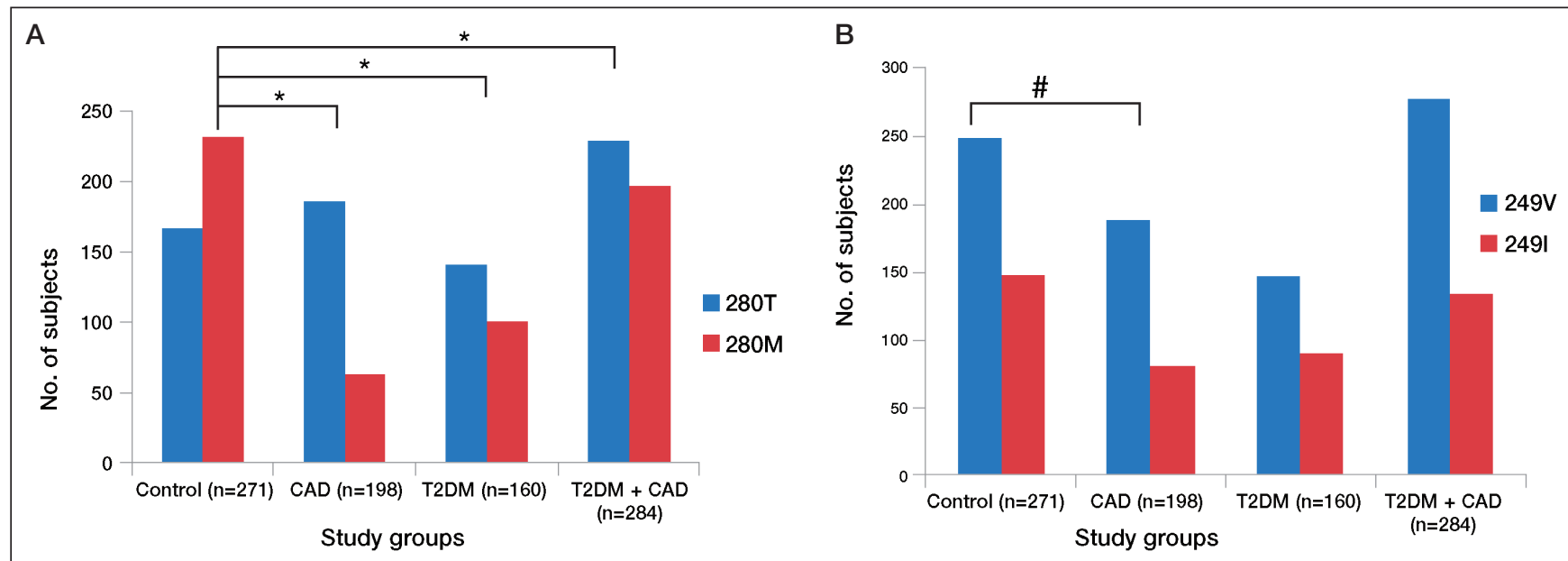

Figure 3) Bar diagram showing allelic distribution of CX3CR1 gene polymorphisms in study groups. A Allelic distribution of T280M polymorphism: M allele is less prevalent compared with the $T$ allele in the disease groups compared with normal healthy subjects $(P<0.01)$. B Allelic distribution of V249I polymorphism: I allele is less prevalent compared with $V$ allele in all disease groups compared with normal healthy subjects. $t$ test analysis shows that $P<0.05$ in patients with coronary artery disease (CAD), $P=0.33$ in type 2 diabetes mellitus (T2DM) patients and $P=0.07$ in patients with T2DM with CAD. No Number

demonstrated similar results in patients with diabetes mellitus. The authors, therefore, investigated the role of these two gene variants in patients with both diabetes and CAD.

The risk estimates of patients in whom T2DM and CAD coexist are tabulated in Table 5. Regression analysis of T280M polymorphism revealed an OR of 0.71 (95\% CI 0.25 to 2.23) for the TM heterozygous and MM homozygous mutant genotypes compared with TT wild genotype. For V249I polymorphism, an OR of 0.97 (95\% CI 0.53 to 2.17) for the VI heterozygous and II homozygous mutant genotypes versus VV wild genotype was observed. These polymorphisms, when present as pure homozygous mutant genotypes, MM (T280M) and II (V249I), significantly reduced the risk for CAD in patients with diabetes.

Logistic regression analysis was performed after adjusting for age, sex, smoking, alcohol consumption, Hb1Ac and cholesterol covariates in each patient group. Allelic distribution of T280M and V249I polymorphisms of CX3CR1 gene in three patient groups and healthy controls is given in Figure 3. The 280M allele of T280M polymorphism was significantly associated with decreased risk for disease; whereas the reduced risk conferred by 249I allele of V249I was not statistically significant.

\section{DISCUSSION}

We report the atheroprotective effect of CX3CR1 V249I and T280M polymorphisms studied in a South Indian population comprising patients with T2DM, CAD, and both T2DM and CAD. The purpose of the present study was to understand the role of these gene variants in diabetic vascular disease.

The gene variants of CX3CR1 are known to have a protective role in $\mathrm{CAD}$ and atherosclerosis by reducing chemokine-receptor interaction. The CX3CR1 receptor is involved in monocyte and leukocyte chemoattraction to vascular endothelium and promotes inflammation. Gene variants in this receptor are believed to attenuate this effect. The soluble form of FKN ligand-CX3CR1 signalling regulates crucial physiological functions necessary for immune regulation by attracting monocytes, natural killer (NK) cells and T lymphocytes. FKN bound to endothelial cell membrane mediates the extravasation of CX3CR1 receptor expressing monocytes, $\mathrm{T}$ cells and $\mathrm{NK}$ cells from blood into the tissue. Membrane-bound FKN interacts with CX3CR1 on leukocytes and mediates rapid and firm adhesion of leukocytes $(11,12)$. Leukocyte infiltration of endothelial cells is mediated by FKNCX3CR1 interaction results in various vascular impairments.

We found that the V249I and T280M gene variants were associated with a reduced risk for CAD. Our results are similar to the findings of
TABLE 5

Frequency of genotypes in controls, and patients with type 2 diabetes mellitus (T2DM) and coronary artery disease (CAD)

\begin{tabular}{lcccc}
\hline Genotype & $\begin{array}{c}\text { Control } \\
(\mathbf{n = 2 7 1 )}\end{array}$ & $\begin{array}{c}\text { T2DM+CAD } \\
(\mathbf{n = 2 8 4})\end{array}$ & OR (95\% Cl) & $\begin{array}{c}\text { Adjusted OR } \\
(\mathbf{9 5 \%} \text { Cl)* }\end{array}$ \\
\hline CX3CR1 T280M & & & \\
T/T & $39(14.4)$ & $88(31)$ & 1 (reference) & 1 (reference) \\
T/M & $127(46.9)$ & $141(49.6)$ & $0.49(0.31-0.76)$ & $1.00(0.33-3.34)$ \\
M/M & $105(38.7)$ & $55(19.4)$ & $0.23(0.14-0.38)$ & $0.44(0.12-1.50)^{\dagger}$ \\
T/M + M/M & $232(85.6)$ & $196(69)$ & $0.37(0.24-0.57)$ & $0.71(0.25-2.23)$ \\
CX3CR1 V249I & & & \\
V/V & $124(45.8)$ & $151(53.2)$ & 1 (reference) & 1 (reference) \\
V/I & $124(45.8)$ & $126(44.4)$ & $0.83(0.59-1.17)$ & $1.09(0.48-2.47)$ \\
I/I & $23(8.5)$ & $7(2.5)$ & $0.24(0.10-0.60)$ & $0.19(0.00-1.87)^{\dagger}$ \\
V/I + I/I & $147(54.2)$ & $133(46.8)$ & $0.74(0.53-1.03)$ & $0.97(0.53-2.17)$ \\
\hline
\end{tabular}

Data presented as $n$ (\%) unless otherwise indicated. *Logistic regression analysis adjusted for age, sex, cholesterol, glycated hemoglobin, smoking and alcohol. ${ }^{\dagger} P<0.05$

McDermott et al (6) and Moati et al (7). MM homozygous genotype of T280M polymorphism in CX3CR1 gene in some cohorts is reported to reduce the risk of acute coronary events (7) and coronary artery endothelial cell dysfunction (6). In contrast to the protective role of these variants in $\mathrm{CAD}$ and atherosclerosis, they are associated with a higher risk for developing systemic sclerosis-associated pulmonary arterial hypertension (13) and ischemic stroke $(14,15)$.

The exact functional effects of the CX3CR1 280M and 249I alleles are unclear. The binding affinity of FKN chemokine ( ${ }^{125}$ Iodine labelled) to cells from CX3CR1-249I-280M homozygotes diminishes in comparison with its binding to wild-type CX3CR1-249I-280M cells (16). Both polymorphisms also result in 'loss-of-function' because the binding sites in cells was found to be significantly reduced in CX3CR1249I-280M homozygotes compared with wild-type controls. In contrast, peripheral blood monocytes from individuals carrying the CX3CR1-249I-280M haplotype attach more to membrane-bound FKN than peripheral blood mononuclear cells from wild CX3CR1V249I-280M donors, indicating a 'gain-of-function' (17).

Recently, there has been renewed interest in the CX3CR1 gene with the discovery of its role in the pancreatic $\beta$-cell insulin secretory pathway (8). With their comprehensive studies using CX3CR1 KO mice islets, Lee et al (8) observed that attenuation of FKN/CX3CR1 system underlie beta cell dysfunction and insulin secretion. The 280M and 249I 
variants alter ligand-receptor binding affinity and are believed to influence an increased susceptibility to T2DM in the carrier. This assumption agrees, in part, with earlier studies by Shah et al (5), involving patients with T2DM, and Sirois-Gagnon et al (18) in obese individuals. They reported a positive association of CX3CR1 polymorphisms in their study subjects. This is, however, in sharp contrast to our findings. In our study, these two variants reduced the risk for T2DM. Homozygosity of mutant CX3CR1 alleles was more frequent in controls than cases. The 249I and 280M alleles were notably associated with the decreased risk for T2DM. Our results cannot be compared with studies investigating obesity (18) because we focused on T2DM and its associated macrovascular complications.

There is a significant paucity of data involving humans to further illustrate these contrasting evidences. The study by Mohan et al (19) involving 2095 subjects with T2DM (the only study involving humans) had an OR of 1.20 with a modest test of significance of 0.05 after adjusting for variables such as age, sex and smoking lifestyle status. The Indian population is more prone to diabetes and its complications. Various studies have reported this trend. It is probable that the disparity in allelic frequency is due to the interethnic differences between the South Indian Dravidian population analyzed in the present study and Caucasian-European populations in earlier studies.

These independent findings in CAD and T2DM prompted us to investigate the significance of CX3CR1 gene variants in patients with vascular diseases associated with diabetes mellitus. We analyzed 284 patients with T2DM diagnosed with CAD for the prevalence of these two genetic variants. The heterozygous TM and homozygous mutant MM genotypes of T280M variants were less prevalent in all patient groups. We report here, for the first time, that $280 \mathrm{M}$ genetic variants of CX3CR1 are also significantly associated with decreased risk for CAD in patients with T2DM. Diabetes mellitus and vascular inflammation leading to $\mathrm{CAD}$ is a multifactorial process involving various

\section{REFERENCES}

1. Ryden L, Standl E, Bartnik M, et al. Guidelines on diabetes, prediabetes, and cardiovascular diseases: Executive summary. The Task Force on Diabetes and Cardiovascular Diseases of the European Society of Cardiology (ESC) and of the European Association for the Study of Diabetes (EASD). Eur Heart J 2007;28:88-136.

2. Chiha M, Njeim M, Chedrawy EG. Diabetes and coronary heart disease: A risk factor for the global epidemic. Int J Hypertens 2012;2012:697240.

3. Scheuner MT. Genetic predisposition to coronary artery disease. Curr Opin Cardiol 2001;16:251-60.

4. Watkins H, Farrall M. Genetic susceptibility to coronary artery disease: From promise to progress. Nat Rev Genet 2006;7:163-73.

5. Shah R, Hinkle CC, Ferguson JF, et al. Fractalkine is a novel human adipochemokine associated with type 2 diabetes. Diabetes 2011;60:1512-8.

6. McDermott DH, Halcox JP, Schenke WH, et al. Association between polymorphism in the chemokine receptor CX3CR1 and coronary vascular endothelial dysfunction and atherosclerosis. Circ Res 2001;89:401-7.

7. Moatti D, Faure S, Fumeron F, et al. Polymorphism in the fractalkine receptor CX3CR1 as a genetic risk factor for coronary artery disease. Blood 2001;97:1925-8.

8. Lee YS, Morinaga H, Kim JJ, et al. The fractalkine/CX3CR1 system regulates beta cell function and insulin secretion. Cell 2013;153:413-25.

9. Ramachandran A, Snehalatha C, Viswanathan V, Viswanatha M, Haffner SM. Risk of non insulin dependent diabetes mellitus conferred by obesity and central adiposity in different ethnic groups: A comparative analysis between Asian Indians, Mexican Americans and Whites. Diabetes Res Clin Pract 1997;36:121-5.

10. Anup KN, Divya S, Harish K, Anilkumar G. Association analysis of common variants in $\mathrm{FOXO} 3$ with type 2 diabetes in a South Indian Dravidian population. Gene 2012 491:182-6.

11. Nishimura M, Umehara H, Nakayama T, et al. Dual functions of fractalkine/CX3C ligand 1 in trafficking of perforin+/granzyme B+ cytotoxic effector lymphocytes that are defined by CX3CR1 expression. J Immunol 2002;168:6173-80.

12. Haskell CA, Cleary MD, Charo IF. Molecular uncoupling of fractalkine-mediated cell adhesion and signal transduction: Rapid flow arrest of CX3CR1-expressing cells is independent of G-protein activation. J Biol Chem 1999;274:10053-8. chemokines (20), scavenger receptors (20), monocytes, endothelial cells, vascular smooth muscle cells $(22,23)$ and growth factors $(24,25)$, which function in disarray, leading to the diabetic vascular disease phenotype. Polymorphisms in CX3CR1 result in reduced chemokine binding. The reduced FKN-CX3CR1 signalling would possible be a compensatory mechanism to prevent the onset of vascular complications in diabetes.

\section{CONCLUSIONS}

The present study demonstrated a positive association for $280 \mathrm{M}$ allele of CX3CR1 gene conferring a protective role for diabetic vascular disease phenotype. The decreased binding of CX3CR1 with its ligand FKN leads to the attenuated interaction between monocytes and vascular endothelium. At the same time, the reduced binding due to these variants did not have a detrimental effect that led to a diabetic phenotype. This is plausible because altered CX3CR1 signalling may reduce adipose inflammation due to its altered chemokine activity.

DISCLOSURES: The authors have no financial disclosures or conflicts of interest to declare.

AUTHOR CONTRIBUTIONS: SS, SR and CCK conceived and designed the experiments, MMP, SS and SR performed the experiments, VRK and ATN performed statistical analysis of data, VRK, AM, CCK provided reagents, materials, analysis tools, SS and SR Prepared manuscript, AM and CCK critical review of manuscript. All authors read and approved the final manuscript.

ACKNOWLEDGEMENTS: The authors thank Professor M Radhakrishna Pillai, Director, Rajiv Gandhi Centre for Biotechnology, Thiruvananthapuram for providing funding and facilities for conducting this study. They also thank Dr NS Pratapchandran and Dr KR Santosh for providing patient samples.

13. Marasini B, Cossutta R, Selmi C, et al. Polymorphism of the fractalkine receptor CX3CR1 and systemic sclerosis-associated pulmonary arterial hypertension. Clin Dev Immunol 2005;12:275-9.

14. Lavergne E, Labreuche J, Daoudi M, et al. Adverse associations between CX3CR1 polymorphisms and risk of cardiovascular or cerebrovascular disease. Arterioscler Thromb Vasc Biol 2005;25:847-53.

15. Hattori H, Ito D, Tanahashi N, et al. T280M and V249I polymorphisms of fractalkine receptor CX3CR1 and ischemic cerebrovascular disease. Neurosci Lett 2005;374:132-5.

16. Faure S, Meyer L, Costagliola D, et al. Rapid progression to AIDS in $\mathrm{HIV}+$ individuals with a structural variant of the chemokine receptor CX3CR1. Science 2000;287:2274-7.

17. Daoudi M, Lavergne E, Garin A, et al. Enhanced adhesive capacities of the naturally occurring Ile249-Met280 variant of the chemokine receptor CX3CR1. J Biol Chem 2004;279:19649-57.

18. Sirois-Gagnon D, Chamberland A, Perron S, Brisson D, Gaudet D, Laprise C. Association of common polymorphisms in the fractalkine receptor (CX3CR1) with obesity. Obesity (Silver Spring) 2010;19:222-7.

19. Mohan V, Sandeep S, Deepa R, Shah B, Varghese C. Epidemiology of type 2 diabetes: Indian scenario. Indian J Med Res 2007;125:217-30.

20. Tretjakovs P, Jurka A, Bormane I, et al. Relation of inflammatory chemokines to insulin resistance and hypoadiponectinemia in coronary artery disease patients. Eur J Intern Med 2009;20:712-7.

21. Handberg A, Levin K, Hojlund K, Beck-Nielsen H. Identification of the oxidized low-density lipoprotein scavenger receptor CD36 in plasma: A novel marker of insulin resistance. Circulation 2006;114:1169-76.

22. Tousoulis D, Charakida M, Stefanadis C. Endothelial function and inflammation in coronary artery disease. Heart 2006;92:441-4.

23. Creager MA, Luscher TF, Cosentino F, Beckman JA. Diabetes and vascular disease: pathophysiology, clinical consequences, and medical therapy: Part I. Circulation 2003;108:1527-32.

24. Kim WJ, Kim SS, Lee HC, et al. Association between serum fibroblast growth factor 21 and coronary artery disease in patients with type 2 diabetes. J Korean Med Sci 2015;30:586-90.

25. Barutcuoglu B, Basol G, Cakir Y, et al. Fibroblast growth factor-19 levels in type 2 diabetic patients with metabolic syndrome. Ann Clin Lab Sci 2011;41:390-6 